\title{
Alasdair Maclntyre: introducción narrativa a su obra
}

\author{
(Alasdair Maclntyre: \\ narrative introduction to his work)
}

\section{HERNANDO JOSÉ BELLO RODRÍGUEZ}

Facultad Eclesiástica de Filosofía, Universidad de Navarra

hernandojose93@gmail.com

ORCID: 0000-0001-9799-8521

\section{JOSÉ MANUEL GIMÉNEZ AMAYA}

Grupo Ciencia, Razón y Fe (CRYF) y Facultad Eclesiástica de Filosofía,

Universidad de Navarra

jmgimenezamaya@unav.es

ORCID: 0000-0002-6977-3963

Resumen. A raíz de la publicación del último libro del filósofo británico Alasdair MacIntyre, Ethics in the Conflicts of Modernity: An Essay on Desire, Practical Reasoning, and Narrative, este trabajo propone una manera de comprender su producción bibliográfica en el contexto de la narrativa de su trayectoria vital e intelectual. Se elabora una periodización del itinerario de MacIntyre, basada en las referencias que el propio autor ha hecho sobre su vida académica y en las propuestas de varios estudiosos de su pensamiento. Se concluye que el examen narrativo de la obra de Alasdair MacIntyre revela que su proyecto filosófico está marcado básicamente por Aristóteles y Tomás de Aquino, cuyo pensamiento ayudó a nuestro autor a articular una propuesta sólida sobre las principales cuestiones de filosofía moral y la filosofía de la religión, y a elaborar un nuevo marco de interpretación y conocimiento sapiencial de la realidad, también en su aspecto científico. Este trabajo se concibe como introductorio porque se centra 
solamente en los libros de Maclntyre, pero se espera que sirva como base sólida para realizar un estudio crítico minucioso - sobre todo, desde una perspectiva cronológicade la práctica totalidad de su obra, que incluye también capítulos de libros, artículos académicos, entrevistas, entre otros.

Palabras clave: Aristóteles; Ética; Filosofía de la religión; Filosofía moral; Proyecto «After Virtue»; Tomás de Aquino.

\begin{abstract}
In the wake of the publication of the last book of the British philosopher Alasdair Maclntyre, Ethics in the Conflicts of Modernity: An Essay on Desire, Practical Reasoning, and Narrative, this work proposes a way to understand his bibliographical production in the context of the narrative of his life and his intellectual career. It is elaborated a periodization of the itinerary of Maclntyre, based on the references that the author himself has made of his academic life and in the proposals of several scholars of his thought. It is concluded that the narrative examination of Maclntyre's work reveals that his philosophical project is basically marked by Aristotle and Aquinas, whose thought helped our author to articulate a solid proposal on the main issues of moral philosophy and philosophy of religion, and to elaborate a new framework of interpretation and sapiential knowledge of reality, including its scientific dimension. This work is conceived as introductory because it focuses only on Maclntyre's books, but it's expected to serve as a solid basis for a thorough critical study -especially from a chronological perspective- of his entire work, which includes chapters of books, papers, interviews, among others.
\end{abstract}

Keywords: Aristotle; Ethics; Philosophy of Religion; Moral Philosophy; «After Virtue» Project; Thomas Aquinas.

En su último libro publicado, Ethics in the Conflicts of Modernity, el filósofo británico Alasdair MacIntyre argumenta que en la medida en que cada uno se mueve hacia su fin último representa - dramatiza - una historia. En este sentido, «nuestras vidas tienen una estructura narrativa» $\mathrm{y}$ «solo podemos hacernos a nosotros mismos inteligibles adecuadamente en términos narrativos» (MacIntyre 2016, 229). Sin duda, Ethics in the Conflicts of Modernity manifiesta la importancia que MacIntyre otorga a la comprensión narrativa de la vida humana.

Por eso, nos parece que no resulta inapropiado intentar comprender la obra completa del filósofo británico desde un punto de vista narrativo. Esta labor exige, a nuestro juicio, una doble tarea. Por una parte, situar cada uno de los libros publicados por el filósofo escocés en el contexto de su historia 
personal y de su trayectoria intelectual; por otra, sugerir de qué manera cada uno de ellos contribuye a su proyecto vital y filosófico. Así aparecerá ante nuestros ojos una narrativa de la obra publicada por MacIntyre.

Este trabajo se centra en los libros de Alasdair MacIntyre: ya sea su autor, co-autor o editor. Se deja de lado, por tanto, una vastísima cantidad de artículos académicos, reseñas, entrevistas e intervenciones en medios de comunicación. Por supuesto, estas otras publicaciones del filósofo británico son importantes. No obstante, nos parece que su estudio crítico se puede abordar mejor si antes está elaborado el marco narrativo de sus libros.

\section{Raíces: formación y aprendizaje (1949-1971)}

En una entrevista concedida a la revista Cogito en 1991, Alasdair MacIntyre dividió su trayectoria intelectual en tres etapas:

Mi vida como filósofo académico se divide en tres partes. [1] Los veintidós años desde 1949, cuando me convertí en estudiante de máster de filosofía en la Universidad de Manchester, hasta 1971 fueron un período, tal y como aparecen ahora retrospectivamente, de investigaciones heterogéneas, mal organizadas, algunas veces fragmentadas y con frecuencia frustrantes y confusas, de las que, no obstante, al final aprendí mucho. [2] Desde 1971, poco después de que emigré a Estados Unidos, hasta 1977 fue un período provisional, en ocasiones de una reflexión autocrítica dolorosa, reforzada por haber llegado a términos críticos con perspectivas tan diferentes en filosofía moral como las ofrecidas por Davidson, por una parte, y Gadamer, por la otra. [3] De 1977 en adelante, he estado comprometido con un solo proyecto del que After Virtue, Whose Justice? Which Rationality? y Three Rival Versions of Moral Enquiry son centrales (Pyle 1999, 77).

En este apartado nos centraremos en la primera etapa. Lo hemos titulado «Raíces: formación y aprendizaje» puesto que aborda los primeros pasos de MacIntyre en la filosofía. En concreto, al usar la palabra «raíces» seguimos la terminología utilizada por otros autores (D’Andrea 2006; Giménez Amaya y Sánchez Migallón 2011; González Pérez 2006). Con este vocablo queremos reflejar que la posterior reorientación del itinerario intelectual de MacIntyre no supone una ruptura total con su pensamiento anterior. 
En 1949, el joven Alasdair MacIntyre, de 20 años, comenzó un Master of Arts en filosofía, en la Universidad de Manchester. Ese mismo año se había graduado de Estudios Clásicos (Bachelor of Arts in Classics) en el Queen Mary College, de la Universidad de Londres. Durante sus años en Queen Mary, de 1945 a 1949, MacIntyre había desarrollado cierto interés por la filosofía, que se manifestaba, por ejemplo, en su asistencia a conferencias de filósofos reconocidos como Alfred Ayer y Karl Popper (MacIntyre 2013, 17). De allí que se trasladara al único lugar donde había un máster en filosofía -Manchester- para proseguir sus estudios (D’Andrea 2006, xvii).

MacIntyre terminó el máster en 1951 con una tesis titulada «The Significance of Moral Judgments». Fue contratado entonces como profesor de filosofía de la religión en la propia Universidad de Manchester. Dos años más tarde el filósofo británico publicaría su primer libro, Marxism: An Interpretation. En él, expresaba su convicción de que era posible ser marxista y cristiano a la vez, sin incoherencia (MacIntyre 2007b, 20). De esta manera, Marxism: An Interpretation reflejaba, en cierto modo, dos facetas de la vida de MacIntyre: su fe cristiana y su adhesión a la crítica marxista del liberalismo.

Por lo que respecta a lo primero, Alasdair MacIntyre fue bautizado presbiteriano, pero su educación no se ciñó a ninguna denominación cristiana en particular. En su adolescencia se interesó por el catolicismo; sin embargo, algunas dudas impidieron que ingresara en la Iglesia católica. Ya en Queen Mary, se involucró en el Student Christian Movement, un movimiento ecuménico que estaba presente en el college. Durante ese tiempo, se inclinó por el anglicanismo, pero tampoco llegó a formalizar ningún vínculo con la Iglesia anglicana (D’Andrea 2006, xvi-xvii).

Por otra parte, con 18 años, MacIntyre se unió al Partido Comunista británico: le pareció convincente la crítica que hacía de la política liberal inglesa. No obstante, un año después abandonó la formación, advirtiendo las atrocidades del comunismo soviético y que el partido en sí mismo era una organización ineficiente (D’Andrea 2006, xvii). Ahora bien, a través del partido comunista el filósofo británico conoció la obra de Karl Marx: le influyó especialmente El 18 brumario de Luis Bonaparte (MacIntyre 2013, 20). 
En las obras posteriores a Marxism: An Interpretation MacIntyre concentró su atención en el campo que trabajaba entonces: la filosofía de la religión. En 1955 editó junto con Anthony Flew New Essays in Philosophical Theology, una colección de 22 artículos de más de 15 autores. El de nuestro autor se titulaba «Visions»: en él, MacIntyre defiende que la creencia religiosa no está válidamente fundamentada cuando se basa en visiones (MacIntyre 1955, 260). Este ensayo, por otra parte, refleja ya los fundamentos de su filosofía de la religión: la teología de Karl Barth y la filosofía de Ludwig Wittgenstein (Lutz 2009, 18). Dos años después, en 1957, publicó con Stephen Toulmin y Ronald Hepburn Metaphysical Beliefs. La obra tenía tres capítulos, cada uno de los cuales era un ensayo de uno de los autores: el de MacIntyre, «The Logical Status of Religious Belief», concluye en la sutil oposición entre la fe y la filosofía, en concreto la metafísica, en la medida en que la primera está más allá de cualquier argumento racional. Las pretensiones de la metafísica pueden ser destructivas para la religión (MacIntyre 1970b, 200-201).

Después de seis años como profesor en Manchester, el interés de MacIntyre por la filosofía moral - reflejado en el tema de su tesis de máster- lo llevó a trasladarse a la Universidad de Leeds. Allí podría dar clases de ética y no de filosofía y psicología de la religión, como hacía hasta entonces (D’Andrea 2006, xvii). Tras un año en Leeds, nuestro autor publicó The Unconscious: A Conceptual Analysis, un ensayo en el que investiga cómo debe ser justificado el concepto de «inconsciente»: se trata de un estudio sobre el trasfondo teórico del psicoanálisis (MacIntyre 1997, 3). Además, MacIntyre refleja en esta obra su concepción sobre la naturaleza de las teorías y explicaciones científicas (González Pérez 2006, 36). Por otra parte, The Unconscious: A Conceptual Analysis fue el primer libro del filósofo británico no editado por SCM Press (SCM son las siglas del ya mencionado Student Christian Movement): lo editó Routledge \& Kegan Paul.

La atracción de MacIntyre por la ética no le impidió seguir investigando en filosofía de la religión. En 1959, todavía en la Universidad de Leeds, sacó a la luz Difficulties in Christian Belief. En este libro, el filósofo británico expresa su «insatisfacción» respecto a la explicación cristiana de algunos temas como la libertad humana y la existencia del mal (González Pérez 
2006, 30) y argumenta que la moral cristiana no puede ser justificada filosóficamente (Lutz 2009, 18-19).

En 1961, tras diez años de docencia universitaria, MacIntyre se fue al Nuttfield College de Oxford para dedicarse a la investigación sociológica: en Leeds se había convencido de que no se puede comprender adecuadamente la ética sin saber sociología (D’Andrea 2006, xvii-xviii). El filósofo británico obtuvo entonces otro Master of Arts (Hull 2013, 309). En 1965, editó Hume's Ethical Writings, una selección de textos sobre ética del filósofo empirista escocés. En la introducción, MacIntyre enmarca los escritos éticos de Hume en el contexto de su obra y de su vida, a la vez que aporta una cierta crítica de sus presupuestos (MacIntyre 1965, 15-16).

Después de cinco años en Oxford - dos en el Nuttfield College y tres en el University College (con algunas estadías en Princeton)-, MacIntyre se desplazó a la Universidad de Essex, en 1966. Allí le habían ofrecido dar clases de sociología; además, podría establecer conversaciones regulares con expertos en las ciencias sociales (D’Andrea 2006, xviii). El mismo año de su llegada a Essex publicó A Short History of Ethics, un libro en el que quedó de manifiesto lo importante que era para el filósofo británico abordar la ética desde una perspectiva histórica (MacIntyre 1998, xxi). En 1967 MacIntyre publicó Secularization and Moral Change, un libro en el que, tal y como sugiere su título, confluían sus dos ámbitos de estudio: la filosofía de la religión y la ética. En esta obra - recopilación de sus Riddell Memorial Lectures, pronunciadas en la Universidad de Newcastle upon Tyne en 1964-, MacIntyre se hace, básicamente, tres preguntas: ¿Por qué la secularización no ha avanzado más de hasta donde ha llegado?, ¿es la decadencia religiosa una o la causa de la decadencia moral?, y ¿qué efecto ha tenido la secularización en el cristianismo inglés? (MacIntyre 1967, 7). Nuestro autor llegará a la conclusión de que la ética cristiana ha llegado a ser incompatible con los cambios sociales que han traído consigo la industrialización y la urbanización, que han propiciado a su vez la secularización (González Pérez 2006, 31). Por tanto, ha sido un cambio social y moral el que ha causado la secularización, no al revés (D’Andrea 2006, 149). 
La aparición de Marxism and Christianity en 1968 -revisión de Marxism: An Interpretation - puso en evidencia que MacIntyre no había conseguido articular coherentemente la crítica marxista y la fe cristiana: «No sabía cómo hacer las paces ni con el marxismo ni con el cristianismo» (MacIntyre 1995, 23). Con respecto al marxismo, después de abandonar el partido comunista, MacIntyre perteneció a dos grupos trotskistas y participó en varios medios de comunicación afines al pensamiento de Marx. Sin embargo, fue saliendo de todos ellos: cada vez estaba más desilusionado con todas las formas de política marxista (Knight 1998, 2).

Por otra parte, respecto a la creencia religiosa (cristiana), resalta la publicación en 1969 de The Religious Significance of Atheism. Esta obra recoge las Bampton Lectures de 1966 pronunciadas por MacIntyre y por Paul Ricoeur. Las de nuestro autor llevan por título «The Debate about God: Victorian Relevance and Contemporary Irrelevance». Allí, MacIntyre sostiene - como lo había hecho en Secularization and Moral Change- que no se trata tanto de que la pérdida de la fe haya producido una pérdida de la moral -como defendería, por ejemplo, Dostoievski-, sino de lo inverso: que un cambio en el carácter de la moral ha provocado en parte que en la modernidad no se acepten las creencias teístas (MacIntyre 1969, 39).

El inicio de la década de los setenta supuso un cambio de contexto en la trayectoria intelectual de MacIntyre. En 1970 el filósofo británico abandonó el mundo universitario británico y se trasladó a Estados Unidos; en concreto a la Universidad de Brandeis, en Waltham, Massachusetts. Ese mismo año se publicaron tres libros de nuestro autor: la segunda edición de Metaphysical Beliefs; Sociological Theory and Philosophical Analysis, editado con Dorothy Emmet (quien había supervisado su trabajo de fin de máster en Manchester); y Herbert Marcuse: An Exposition and a Polemic. Cada uno de estos libros aborda diversas líneas de investigación filosófica que hasta ahora había seguido MacIntyre: la filosofía de la religión, la sociología y el marxismo, respectivamente. En la segunda edición de Metaphysical Beliefs, el filósofo británico reconoce -en el nuevo prefacio-que el irracionalismo presente en su ensayo es «falso y peligroso» (MacIntyre 1970a, xi). En la introducción de Sociological Theory and Philosophical Analysis Emmet 
y MacIntyre desarrollan su visión sobre la relación entre la filosofía y las ciencias sociales; afirman, por ejemplo, que no se trata de adoptar una determinada filosofía y aplicarla a la reflexión sobre las ciencias sociales; sino de discernir en qué medida una corriente filosófica ayuda a resolver los principales problemas teóricos de dichas ciencias (Emmet y MacIntyre 1970, ix). Por último, en Herbert Marcuse: An Exposition and a Polemic MacIntyre manifiesta su rechazo al marxismo de Marcuse, por considerar que se trata de una concepción elitista de reforma social (D’Andrea 2006, 116).

Después de un año en Brandeis, en 1971, MacIntyre publicó Against the Self-Images of the Age: Essays on Ideology and Philosophy, libro que cierra esta primera etapa de la narrativa de la obra del filósofo británico. Él mismo lo cuenta de esta manera:

Cualquier narración adecuada de mi vida tendría que enfatizar un cambio radical alrededor de 1971. Hasta entonces, yo había tenido una serie de conjuntos de intereses y creencias dispares y en ocasiones conflictivos, y era incapaz de moverme decisivamente hacia cualquier solución, ya sea de los problemas internos a cada conjunto particular de intereses y creencias, o de los problemas que surgían de la tensión entre ellos [...]. Los ensayos recopilados en Against the Self-Image of the Age (1971) cerraron este período (Pyle 1999, 76-77).

Los ensayos de Against the Self-Images of the Age reflejan que, para el MacIntyre de entonces, el cristianismo, el psicoanálisis y sobre todo el marxismo habían fracasado tanto intelectualmente como a la hora de expresar las formas de pensar y actuar de la vida social contemporánea: la capacidad de comprender mejor la sociedad actual dependía, más bien, de las investigaciones en filosofía moral y en filosofía de las ciencias sociales (MacIntyre 1978, viii-ix).

\section{2. «Reflexión autocrítica dolorosa» (1971-1977)}

Algunos de los últimos libros publicados por MacIntyre hasta 1971 reflejaban cierto espíritu revisionista de sus primeros trabajos. Los primeros años en Estados Unidos supusieron para el filósofo británico un período de «reflexión autocrítica dolorosa» (Pyle 1999, 77). 
En 1972, en apariencia ajeno a sus anteriores libros, MacIntyre editó Hegel: A Collection of Critical Essays. Esta colección de ensayos se proponía desmitificar la figura del Hegel metafísico racionalista y recuperarlo como un filósofo viviente preocupado con asuntos genuinos (MacIntyre 1972, viii). El ensayo de nuestro autor, titulado «Hegel on Faces and Skulls», reivindica la importancia de la comprensión histórica del ser humano, frente a una mera comprensión teórico-científica (MacIntyre 1972, 235-236): la insistencia en la comprensión histórica ya se había percibido en A Short History of Ethics.

El mismo año de la aparición del libro sobre Hegel, MacIntyre dejó la Universidad de Brandeis y se trasladó a la Universidad de Boston. Sus primeros cinco años allí, de 1972 a 1977, transcurrieron sin que el filósofo británico publicara un libro. Escribió, eso sí, una decena capítulos para libros editados por otros autores, además de varios artículos académicos, reseñas e intervenciones en medios de comunicación.

En 1977 culminó, según lo narra el propio MacIntyre, el período de «reflexión autocrítica dolorosa». Ese mismo año contrajo matrimonio con Lynn Sumida Joy, una estudiante de posgrado de Harvard (Hull 2013, 311; Sayre 2014, 244). No son pocos los libros posteriores de MacIntyre que incluirán alguna referencia (casi siempre en el prólogo) a Joy.

\section{El proyecto «After Virtue» (1977-actualidad)}

El título de este apartado lo tomamos prestado de otros autores (D’Andrea 2006; Giménez Amaya y Sánchez Migallón 2011; González Pérez 2006). Con él nombramos la tercera etapa de la narrativa de la obra de MacIntyre, que el mismo filósofo británico sitúa a partir de 1977.

Se habla del proyecto «After Virtue» porque esta fue el primer libro publicado por Alasdair MacIntyre tras 1977. Como en este trabajo solo nos centramos en los libros de MacIntyre, apenas podemos apuntar la importancia de su artículo «Epistemological Crises, Dramatic Narrative and the Philosophy of Science», publicado en la revista The Monist, precisamente en 1977. Queda pendiente explicar en qué medida este artículo manifiesta la transición entre dos etapas de la trayectoria intelectual del filósofo 
británico (en una colección de ensayos de la que hablaremos más adelante, The Tasks of Philosophy, MacIntyre incluirá este artículo. Véase MacIntyre 2006a, vii-viii y 3-23).

After Virtue salió a la luz en 1981. MacIntyre era entonces profesor del Wellesley College, donde había sido contratado el año anterior como el primer Henry R. Luce, Jr. Professor of Language, Mind and Culture. En 1979 había dejado la Universidad de Boston y se había trasladado a la Universidad de Princeton, en la que estuvo un año.

MacIntyre había comenzado a escribir After Virtue en 1972 (Yepes Stork 1990, 87), con lo cual trabajó en esta obra casi una década. Este libro se incluye claramente entre aquellos en los que el filósofo británico se centra en la ética. Se caracteriza por su marcada inspiración aristotélica y su rechazo de la modernidad tanto ilustrada como emotivista. Supuso, además, la revitalización del concepto de «virtud» en la filosofía moral (González 2016, 142), aunque MacIntyre ha sostenido en varias ocasiones que él no se considera, sin más, un defensor de la «ética de la virtud» como alternativa a la «ética del deber» o al consecuencialismo (MacIntyre 2013, 29-30).

Al año siguiente de publicar After Virtue, en 1982, MacIntyre dejó el Wellesley College y se trasladó a la Universidad de Vanderbilt, que les había ofrecido tanto a él como a su mujer puestos de trabajo (D’Andrea 2006, p. xviii). En 1983, el filósofo británico editó con Stanley Hauerwas Revisions: Changing Perspectives in Moral Philosophy. En el prefacio de este libro los autores muestran su sospecha ante el entonces auge de la disciplina de la ética, porque para ellos era signo del debilitamiento de los vínculos morales de la sociedad (Hauerwas y MacIntyre 1983, vii). Por lo que respecta a MacIntyre, su preocupación -ya expresada con fuerza en After Virtue - radicaba en el gran desacuerdo existente en la moral y en la filosofía moral contemporánea, posible señal de la carencia de recursos en la cultura moral actual para resolver tal desacuerdo (MacIntyre 1983, 5).

Por otra parte, el debate que se generó en torno a After Virtue llegó a tales dimensiones que MacIntyre decidió responder a las críticas en una segunda edición del libro, publicada en 1984. En el epílogo, se defendió de las acusaciones que le hacían, grosso modo, de ser historicista, relativista y 
de confundir los planos de la ética y de la teología moral (MacIntyre 2007a, 265-78).

\subsection{Desarrollo del proyecto «After Virtue» (1988-1995)}

Después de seis años en Vanderbilt, en 1988, Alasdair MacIntyre cambió de contexto: ahora su alma máter sería la Universidad de Notre Dame. Ese mismo año publicó Whose Justice? Which Rationality?.

Este libro puede ser considerado la continuación de las investigaciones filosóficas que MacIntyre comenzó con After Virtue. En esta última obra, el filósofo británico había prometido ofrecer una explicación de qué hace que sea racional actuar de una determinada manera; incluso de qué hace que sea racional defender una determinada concepción de racionalidad práctica. Whose Justice? Which Rationality? viene a ser el cumplimiento de esa promesa (MacIntyre 1988, ix). Por otra parte, este texto refleja que MacIntyre había abrazado la filosofía de Tomás de Aquino, ante la cual mostraba ciertas resistencias en After Virtue (MacIntyre 1988, x; Giménez Amaya y Sánchez-Migallón 2011, 108-9).

Dos años después de Whose Justice? Which Rationality?, en 1990 y todavía estando en Notre Dame, salieron a la luz dos obras de MacIntyre: Three Rival Versions of Moral Enquiry y First Principles, Final Ends and Contemporary Philosophical Issues. Ambas continúan el desarrollo del proyecto «After Virtue» y dejan patente que el filósofo británico se había insertado plenamente en la tradición tomista (Giménez Amaya y Sánchez-Migallón 2011, 112).

Three Rival Versions of Moral Enquiry recoge y revisa las Gifford Lectures impartidas por MacIntyre en la Universidad de Edimburgo, en 1988. En ellas, el filósofo británico contrasta tres concepciones de la ética -de la investigación moral- que él considera rivales. Según nuestro autor, cada una en su origen tiene un texto de finales del siglo XIX: una concepción brota de la novena edición de la Enciclopedia Británica; otra, de La genealogía de la moral, de Friedrich Nietzsche; y la última, de la encíclica Aeterni Patris, del Papa León XIII (MacIntyre 1990, 2). MacIntyre advierte la situación conflictiva contemporánea, esta vez en el campo de las humanidades y de 
la investigación universitaria: el conflicto se basa, según él, en las concepciones diferentes de racionalidad (MacIntyre 1990, 7-8). Al final, entre las tres versiones rivales de la investigación moral, nuestro autor opta por la tradición tomista, aquella basada en la encíclica de León XIII (Giménez Amaya y Sánchez-Migallón 2011, 116; González Pérez 2006, 101-102).

Por otra parte, First Principles, Final Ends and Contemporary Philosophical Issues recoge las Aquinas Lectures de MacIntyre en la Universidad de Marquette, en 1990. En esta obra, el filósofo británico muestra la manera como el aristotelismo tomista puede aportar a la hora de comprender las ciencias, por una parte, y los problemas de la filosofía contemporánea, por otra (Giménez Amaya y Sánchez-Migallón 2011, 117). Posteriormente este pequeño libro será recogido en la colección *de ensayos ya mencionada The Tasks of Philosophy (MacIntyre 2006a, 143-78).

Hasta 1995, MacIntyre no volvería a publicar ningún libro.

\subsection{Revisiones (1995-2004)}

El título exacto para este apartado sería uno más largo que diría: «Revisiones a partir del proyecto 'After Virtue' y del propio proyecto 'After Virtue'». Efectivamente, se puede decir que desde los compromisos de este proyecto Alasdair MacIntyre revisa algunas de sus obras anteriores. Y no solo eso: también corrige alguna de las tesis que sostuvo y desarrolló desde la publicación de After Virtue.

En 1995, con 66 años, MacIntyre dejó Notre Dame para enseñar en la Universidad de Duke. Este cambio se debe a que su mujer, Lynn Sumida Joy, también profesora de filosofía, estaba interesada en un departamento con una mayor orientación histórica: Duke satisfacía estas condiciones (D’Andrea 2006, p. xviii).

Ese mismo año, MacIntyre publicó la segunda edición de Marxism and Christianity, con una nueva introducción, titulada «Three Perspectives on Marxism: 1953, 1968, 1995». En ella, el filósofo británico hace una evaluación de sus pretensiones al publicar Marxism: An Interpretation (1953) y la primera edición de Marxism and Christianity (1968). Pero, sobre todo, expone cómo 
gracias a la filosofía de Aristóteles finalmente pudo, por una parte, remover los obstáculos que le impedían abrazar el catolicismo; y, por otra, comprender la verdad de las condenas que hacía la Iglesia católica del marxismo, a la vez que captaba lo que debían aprender los católicos de la crítica marxista: la estrecha relación entre teoría y praxis (MacIntyre 2007b, 27-28).

Al año siguiente, en 1996, salió a la luz la segunda edición de A Short History of Ethics, con un nuevo prefacio de MacIntyre. En él, nuestro autor reconoce explícitamente que si bien está de acuerdo con muchas de las cosas que allí afirma, no obstante su «historia» requiere ser revisada (MacIntyre 1998, viii). De hecho, en el prefacio lleva a cabo la tarea de señalar abiertamente qué cosas deben ponerse en cuestión o ser corregidas.

Ahora bien, si hay que destacar alguna publicación de estos años, sin duda sería Dependent Rational Animals: Why Human Beings Need the Virtues, que salió a la luz en 1999. En este libro -que revisa y amplía las Paul Carus Lectures que ofreció MacIntyre en las reuniones de la división del Pacífico de la American Philosophical Association en 1997- el filósofo británico corrige algunas de las investigaciones plasmadas en la trilogía que constituye la piedra angular del proyecto «After Virtue»: el propio After Virtue, Whose Justice? Which Rationality? y Three Rival Versions of Moral Enquiry. Por ejemplo, considera erróneo desvincular ética y biología, tal y como suponía en After Virtue, o pensar que la filosofía de Tomás de Aquino solo complementa la de Aristóteles, cuando en realidad la corrige sustancialmente en algunos puntos (MacIntyre 1999, x-xi).

En el año 2000, MacIntyre regresó a la Universidad de Notre Dame, en concreto, al Center for Ethics and Culture. Los primeros años del siglo XXI no vieron ninguna obra suya, hasta que en 2004 publicó una segunda edición de The Unconscious: A Conceptual Analysis, cuya primera edición había salido 45 años atrás. En el nuevo prefacio, de casi cuarenta páginas, el filósofo británico se propone decir aquello que no fue explicado o explicado brevemente o con inexactitud en la primera edición (MacIntyre 2004, 2). Además, nuestro autor sostiene que él considera complementarias la comprensión psicoanalítica y algunas tesis de Aristóteles o de Agustín de Hipona (MacIntyre 2004, 38). 


\subsection{Evolución del proyecto «After Virtue» (2005-actualidad)}

Después de la edición revisada de The Unconscious: A Conceptual Analysis, que salió a la luz teniendo MacIntyre 75 años, sería lógico pensar que nuestro autor no publicaría nada más. Nada más lejos de lo que después resultó: el filósofo británico siguió produciendo, profundizando en sus investigaciones, e incluso explorando nuevos campos.

Así, en 2005, publicó Edith Stein: A Philosophical Prologue, 1913-1922, un libro en el que, a partir del estudio de Edith Stein, se evidencia la estrecha relación que MacIntyre percibe entre el pensamiento de un filósofo, su trayectoria vital y el contexto práctico y filosófico en el que se encuentra (MacIntyre 2008, 17-25).

Un año después, publica una colección de ensayos en dos volúmenes: The Tasks of Philosophy y Ethics and Politics. El primero de estos libros recoge ensayos que muestran el cambio de enfoque de la filosofía de MacIntyre a partir de 1977 y su postura frente al contexto moderno, además de abordar cuestiones sobre el papel de la filosofía y su relación con la práctica. En el segundo volumen, los ensayos se caracterizan por reflejar, de manera más o menos explícita, la filosofía aristotélico-tomista de nuestro autor (MacIntyre 2006b, vii).

En 2007 aparece la tercera edición de After Virtue con un nuevo prólogo: «After Virtue after a Quarter of a Century». En este, MacIntyre reafirma las principales tesis de la primera edición, pero también apunta aquellas cosas en las que había cambiado; por ejemplo, la adopción de la filosofía de Tomás de Aquino. Además, responde a diversas críticas que se le han hecho: de nostálgico por un pasado ideal, de relativista y de comunitarista (MacIntyre 2007a, viii-xii).

Un par de años más tarde, MacIntyre publicó God, Philosophy, Universities, que como explica el subtítulo del libro es una historia selectiva de la tradición filosófica católica. En la introducción, nuestro autor apunta las tres convicciones que le llevaron a escribir el libro: que los laicos católicos necesitan comprender mejor la filosofía católica; que la filosofía católica se entiende mejor de forma histórica; y que la filosofía no es solo cuestión 
de argumentos, sino que tiene que ver con filósofos en contextos sociales y culturales particulares, que influyen en el debate filosófico (MacIntyre 2009, 1).

En 2010, después de una década en Notre Dame, MacIntyre se retiró de la enseñanza. Sin embargo, continuó y continúa con su labor investigadora, trabajando en el Centre for Contemporary Aristotelian Studies in Ethics and Politics (CASEP), de la Universidad Metropolitana de Londres. A la vez, MacIntyre es Permanent Senior Distinguished Research Fellow del Center for Ethics and Culture de la Universidad de Notre Dame.

Después de poco más de un lustro en el CASEP, en el 2016, se publicó la que es hasta ahora la última obra de Alasdair MacIntyre: Ethics in the Conflicts of Modernity: An Essay on Desire, Practical Reasoning, and Narrative. Podemos considerar este libro como la síntesis y el testamento del pensamiento filosófico de MacIntyre. Allí, nuestro autor expone y defiende su versión aristotélico tomista de la moral, a la que añade algunos apuntes de la crítica marxista y una comprensión narrativa de la vida humana (MacIntyre 2016, xi).

De esta manera, se capta la evolución del proyecto «After Virtue», que, inicialmente aristotélico, acogió y se reconfiguró a partir de la filosofía de Tomás de Aquino; integró las intuiciones acertadas de Marx en su crítica al liberalismo; y encontró en la fórmula de la narrativa la manera de abordar y estudiar de forma conveniente la acción moral.

\section{Conclusiones}

En la introducción de este trabajo se afirmó que comprender de forma narrativa la obra publicada por Alasdair MacIntyre implicaba una doble tarea: por una parte, situar cada uno de sus libros en su trayectoria vital e intelectual y, por otra, apuntar de qué manera contribuían a su proyecto filosófico. Nos parece que la exposición hecha atrás da por cumplida la primera parte de esta doble tarea. Ahora, en las conclusiones, conviene insistir en la segunda parte. 
¿Cuál es el proyecto filosófico de Alasdair MacIntyre? Aquel en el que confluyen dos grandes vertientes - la segunda, con el paso del tiempo, más grande que la primera-que se corresponden con sendas disciplinas filosóficas: la filosofía de la religión y la filosofía moral. El análisis desde un enfoque narrativo de la obra de MacIntyre nos muestra él fue, poco a poco, resolviendo los conflictos que le planteaban los grandes interrogantes de esos dos ámbitos de estudio y cómo, finalmente, encontró en la filosofía de Aristóteles y de Tomás de Aquino la mejor fórmula para responderlos.

La división en tres etapas de la trayectoria de MacIntyre - basada en lo que él mismo ha dicho sobre su vida intelectual- sirve para comprender mejor su proyecto filosófico. Así, en la primera etapa, nos encontramos sus primeros planteamientos sobre la filosofía de la religión y la filosofía moral; en la segunda, una mirada crítica con esos planteamientos; y, en la tercera, la respuesta que MacIntyre ha hallado más convincente para las preguntas de esas disciplinas, que para nuestro autor está -como se ha dicho-en la tradición aristotélico-tomista.

El contenido del último libro de MacIntyre, Ethics in the Conflicts of Modernity, confirma este hecho. Si bien el filósofo británico también propone rescatar algunos elementos de la crítica marxista e insiste en la importancia de la narratividad, esto no opaca su convicción de la verdad de la filosofía aristotélico-tomista. Gracias a ella, MacIntyre pudo articular con solidez su pensamiento filosófico sobre la religión y la moral. Y no solo articularlo, sino también vivirlo.

\section{Referencias}

D’Andrea, Thomas. 2006. Tradition, Rationality, and Virtue: The Thought of Alasdair MacIntyre. Aldershot: Ashgate.

Emmet, Dorothy y Alasdair MacIntyre, eds. 1970. Sociological Theory and Philosophical Analysis. New York: Macmillan.

Giménez Amaya, José M., y Sergio Sánchez-Migallón. 2011. Diagnóstico de la Universidad en Alasdair MacIntyre: Génesis y desarrollo de un proyecto antropológico. Pamplona: Eunsa.

González, Ana M. 2016. La ética explorada. Segunda edición. Pamplona: Eunsa. 
González Pérez, Juan. 2006. Una biografía intelectual de Alasdair MacIntyre. Cuadernos Empresa y Humanismo, 97. Pamplona: Servicio de Publicaciones de la Universidad de Navarra.

Hauerwas, Stanley, y Alasdair MacIntyre, eds. 1983. Revisions: Changing Perspectives in Moral Philosophy. Notre Dame: University of Notre Dame Press.

Hull, Richard. 2013. "Alasdair Chalmers MacIntyre.” En Presidential Addresses of the American Philosophical Association 1981-1990 (volume 9), editado por Richard Hull, 309-12. Tallahassee: RTH.

Knight, Kelvin, ed. 1998. The MacIntyre Reader. Notre Dame: University of Notre Dame Press.

Lutz, Christopher. 2009. Tradition in the Ethics of Alasdair MacIntyre: Relativism, Thomism, and Philosophy. Paperback Edition. Lanham: Lexington Books.

MacIntyre, Alasdair. 1955. "Visions." En New Essays in Philosophical Theology, editado por Antony Flew y Alasdair MacIntyre, 254-60. London: SCM Press.

MacIntyre, Alasdair, ed. 1965. Hume's Ethical Writings. London: Collier Books.

MacIntyre, Alasdair. 1967. Secularization and Moral Change. London: Oxford University Press.

MacIntyre, Alasdair. 1969. “The Debate about God: Victorian Relevance and Contemporary irrelevance.” En The Religious Significance of Atheism, de Alasdair MacIntyre y Paul Ricoeur, 1-55. New York: Columbia University Press.

MacIntyre, Alasdair. 1970a. Prefacio a la segunda edición de Metaphysical Beliefs, de Stephen Toulmin, Ronald Hepburn y Alasdair MacIntyre, vii-xii. London: SCM Press.

MacIntyre, Alasdair. 1970b. “The Logical Status of Religious Belief.” En Metaphysical Beliefs, Second Edition, de Stephen Toulmin, Ronald Hepburn y Alasdair MacIntyre, 157-201. London: SCM Press.

MacIntyre, Alasdair, ed. 1972. Hegel: A Collection of Critical Essays. Garden City: Anchor Books.

MacIntyre, Alasdair. 1978. Against The Self-Images of the Age: Essays on Ideology and Philosophy. Notre Dame: University of Notre Dame Press.

MacIntyre, Alasdair. 1983. “Moral Philosophy: What Next?” En Revisions: Changing Perspectives in Moral Philosophy, editado por Stanley Hauerwas y Alasdair MacIntyre, 1-15. Notre Dame: University of Notre Dame Press.

MacIntyre, Alasdair. 1988. Whose Justice? Which Rationality?. Notre Dame: University of Notre Dame Press.

MacIntyre, Alasdair. 1990. Three Rival Versions of Moral Enquiry: Encyclopaedia, Genealogy, and Tradition. London: Duckworth. 
MacIntyre, Alasdair. 1997. The Unconscious: A Conceptual Analysis. Bristol: Thoemmes Press.

MacIntyre, Alasdair. 1998. A Short History of Ethics: A History of Moral Philosophy from the Homeric Age to the Twentieth Century. Second Edition. London: Routledge.

MacIntyre, Alasdair. 1999. Dependent Rational Animals: Why Human Beings Need the Virtues. Chicago \& La Salle: Open Court.

MacIntyre, Alasdair. 2004. The Unconscious: A Conceptual Analysis. Revised Edition. New York \& London: Routledge.

MacIntyre, Alasdair. 2006a. The Tasks of Philosophy: Selected Essays, Volume 1. Cambridge: Cambridge University Press.

MacIntyre, Alasdair. 2006b. Ethics and Politics Selected Essays, Volume 2. Cambridge: Cambridge University Press.

MacIntyre, Alasdair. 2007a. After Virtue: A Study in Moral Theory. Third Edition. London: Duckworth.

MacIntyre, Alasdair. 2007b. Marxismo y cristianismo. Traducido por Francisco Javier Martínez y Sebastián Montiel. Granada: Editorial Nuevo Inicio.

MacIntyre, Alasdair. 2008. Edith Stein: Un prólogo filosófico, 1913-1922. Traducido por Feliciana Merino Escalera. Granada: Editorial Nuevo Inicio.

MacIntyre, Alasdair. 2009. God, Philosophy, Universities: A Selective History of the Catholic Philosophical Tradition. Lanham: Rowman and Littlefield.

MacIntyre, Alasdair. 2013. “On Having Survived the Academic Moral Philosophy of the Twentieth Century." En What Happened In and To Moral Philosophy in the Twentieth Century?: Philosophical Essays in Honor of Alasdair MacIntyre, editado por Fran O’Rourke, 17-34. Notre Dame: University of Notre Dame Press.

MacIntyre, Alasdair. 2016. Ethics in the Conflicts of Modernity: An Essay on Desire, Practical Reasoning, and Narrative. Cambridge: Cambridge University Press.

Pyle, Andrew, ed. 1999. Key Philosophers in Conversation: The Cogito Interviews. London \& New York: Routledge.

Sayre, Kenneth. 2014. Adventures in Philosophy at Notre Dame. Notre Dame: University of Notre Dame Press.

Yepes Stork, Ricardo. 1990. “Después de Tras la Virtud.” Atlántida 1, 4: 87-95. 